\title{
Paraneoplastic Subacute Necrotic Myelopathy
}

\author{
Adrian Handforth, Sukriti Nag, Daniel Sharp and David M. Robertson
}

\begin{abstract}
SUMMARY: A 79 year old male acutely developed a Brown-Sequard syndrome two weeks following resection of renal cell carcinoma. The subsequent clinical course was marked by subacute progression of spinal cord signs. Pathologic studies showed extensive multifocal spinal cord necrosis that could not be attributed to vascular occlusion or to vasculitis. This case appears to be a rare example of paraneoplastic subacute necrotic myelopathy.
\end{abstract}

RÉSUMÉ: Un homme de 79 ans a subitement développé un syndrome de Brown-Sequard deux semaines après l'exérèse d'un carcinome à cellules rénales. Par la suite on note la progression subaigue des symptômes d'atteinte de la moelle. La pathologie montra de multiples nécroses étendues qui ne peuvent pas être attribuées à une occlusion vasculaire ou à une vasculite. Il semble donc que le présent cas représente un rare exemple de myélopathie nécrosante subaigue paranéoplasique.

Can. J. Neurol. Sci. 1983; 10:204-207

Subacute necrotic myelopathy, occurring in the absence of vascular anomalies, is a rare disorder of obscure etiology which may occur in an idiopathic, a post-infectious or a paraneoplastic setting (Folliss and Netsky 1970). The association with malignancy is rare and several hypotheses for this association have been advanced but the relationship remains as unexplained as when Mancall and Rosales (1964) first reviewed this puzzling entity. We describe a case of necrotizing myelopathy in a man with renal cell carcinoma. To our knowledge an association with this form of malignancy has not previously been described.

\section{Case Report}

A 79 year old man was admitted to hospital on December 14, 1981 , following the onset of weakness of the right leg. Five weeks previously the patient had experienced sudden right flank pain and was admitted ten days later with a tender right abdominal mass. Ultrasound and renal arteriography showed a solid mass, $8.5 \mathrm{~cm}$. in diameter, occupying the lower pole of the right kidney. The vascular pattern was consistent with renal cell carcinoma. A spring coil was placed in the right renal artery in an attempt to occlude the arterial supply of the tumour but occlusion did not occur. The procedure was otherwise uncomplicated. Six days later a radical nephrectomy was performed, and histological study confirmed the diagnosis. Tumour cells were seen within the lumen of one vessel within the mass.

Three days prior to his last admission, and two and a half weeks post-operatively, the patient noted sudden sharp pain radiating from the right hip to the foot associated with numbness and tingling of the leg, quickly followed by weakness so that he was unable to walk. On the day of admission, he developed constipation and urinary retention. The patient was alert. Examination of cranial nerves was unremarkable. Left leg strength was normal. The right leg was hypotonic. The right hip and knee extensors were unable to overcome gravity but right ankle dorsiflexion was only mildly weak. Sensory examination revealed impaired vibration and proprioception on the left side extending up to approximately the T6 level. Muscle stretch reflexes were symmetrical in upper extremities but reduced at the right knee and ankle. Bilateral Babinski signs were present. Sensory and motor examination of the upper extremities was unremarkable.

Lumbar puncture showed clear colourless fluid with two polymorphonuclear leukocytes and two mononuclear cells. The CSF protein was $54 \mathrm{mg} / \mathrm{dl}$. No malignant cells were found on cytologic examination. A myelogram was normal. A CT scan of brain showed cortical atrophy and a questionable hypodense area in the left frontal lobe but no enhancing lesions.

By the third day after admission, weakness in the right leg had progressed so that the toes could not be moved and flexors and extensors of the ankle, knee and hip showed only a flicker of movement or insufficient power to overcome gravity. In addition, the left leg now also displayed diffuse weakness, so that there was inability to overcome gravity by the hip flexors, knee extensors, ankle dorsiflexors and plantar flexors. The left leg showed loss of vibration and position sense.

One week after admission, both legs were totally paralysed and the patient complained of back pain and bilateral arm pain. The patient developed septicaemia and died of a cardiopulmonary arrest two weeks following admission.

\section{Autopsy Findings}

The lungs showed bronchopneumonia. The right renal artery, with coil in place, was thrombosed. Apart from coronary artery stenosis, the heart was unremarkable. Moderate atherosclerosis was present in the thoracic and abdominal aorta. Examination of intercostal arteries revealed these to be patent. No residual tumour was found.

The external surface of the formalin-fixed spinal cord revealed a circumferential area of dark pulp-like softening maximal from the T5 to T9 segments. The major arterial supply to the thoracic cord was via arteries which entered with the T4 root on left and the $\mathrm{T} 10$ root on the right. On sectioning, these vessels were found to be patent. 
Coronal sections of the spinal cord demonstrated that necrosis extended far more rostrally and caudally than was apparent from external examination, and that the zones of necrosis were not contiguous (Figure 1). The largest band of necrosis extended from C8 to L3 segments of the cord (Figure 2). At mid-portions of this zone the segments T5 toT8 showed complete transverse necrosis. Both rostrally and caudally the necrosis narrowed to a core, then extended as finger-like processes, each finger located within the base of a dorsal column (Figure 3). The caudal parts of $\mathrm{L} 3$ and $\mathrm{L} 5$ appeared normal, but in the intervening segments a small band of necrosis was located within the base

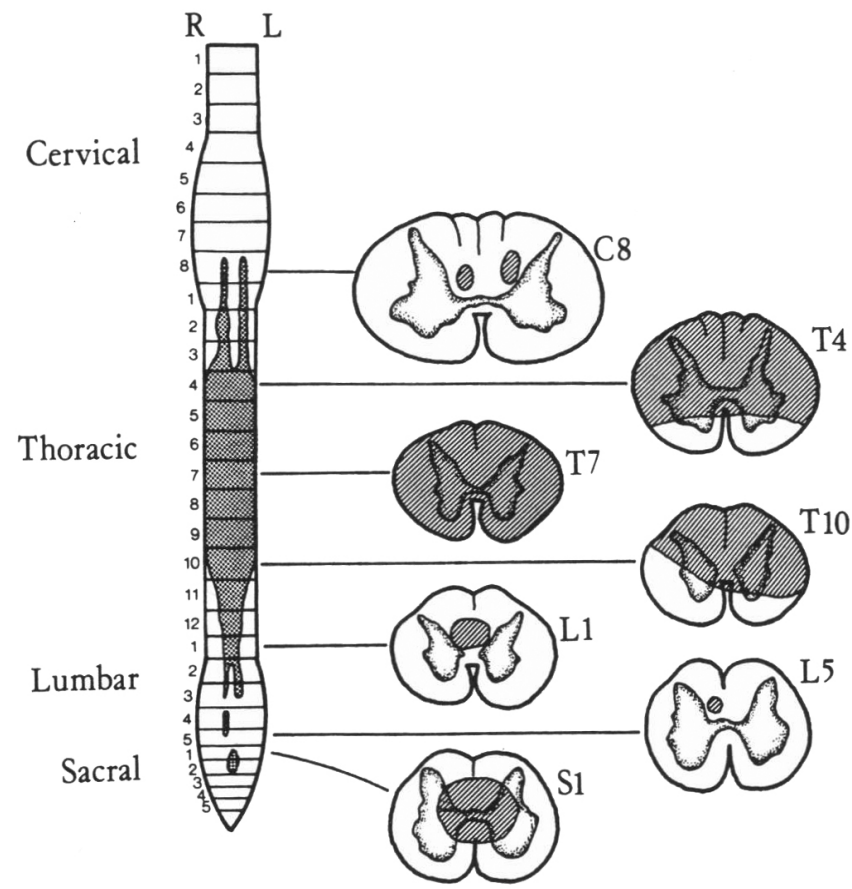

Figure 1-Spinal cord levels showing necrosis, as shown by darkened areas on coronal sections of the fixed cord.

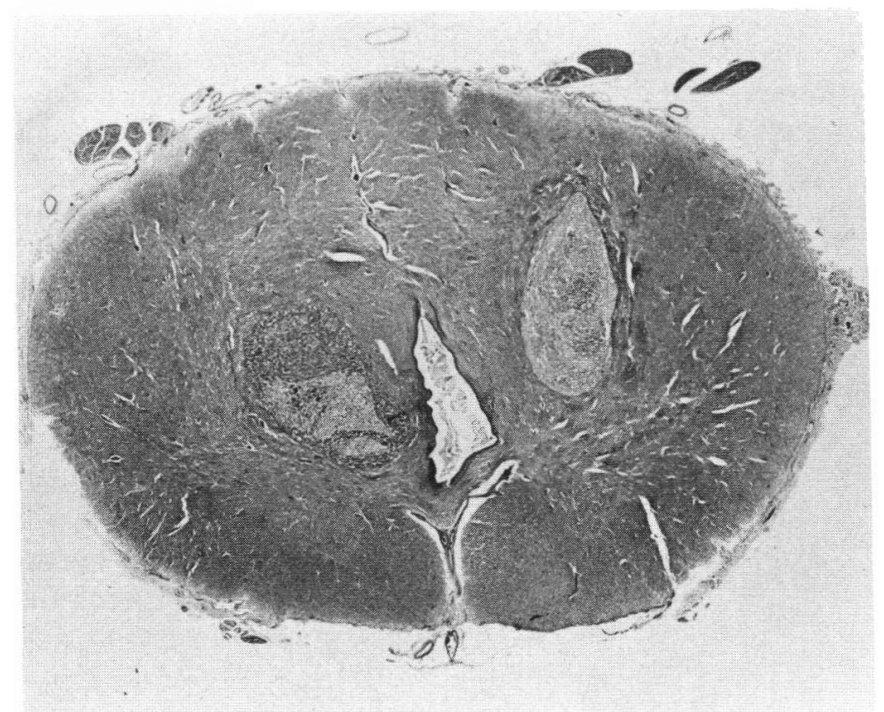

Figure 3 - Spinal cord at first thoracic level, showing a discrete area of necrosis in the base of each dorsal column. An incidental hydromyelia is present. (H\&E-LFB; $x 7.5)$. of the right dorsal column. A larger lesion appeared at the base of the dorsal columns in the rostral part of S1 and expanded to occupy the core of the spinal cord in the lower part of $\mathrm{S} 1$ and in S2 (Figure 4). The remaining sacral segments were normal.

Microscopic sections of spinal cord were stained with hematoxylin and eosin-Luxol fast blue. Areas which were grossly soft and darkened were shown on histologic examination to consist of necrotic tissue with macrophages, extravasated red blood cells, and polymorphonuclear cells. At T7, a level of total destruction of grey and white matter, the lesion appeared more advanced in that relatively more macrophages with ingested

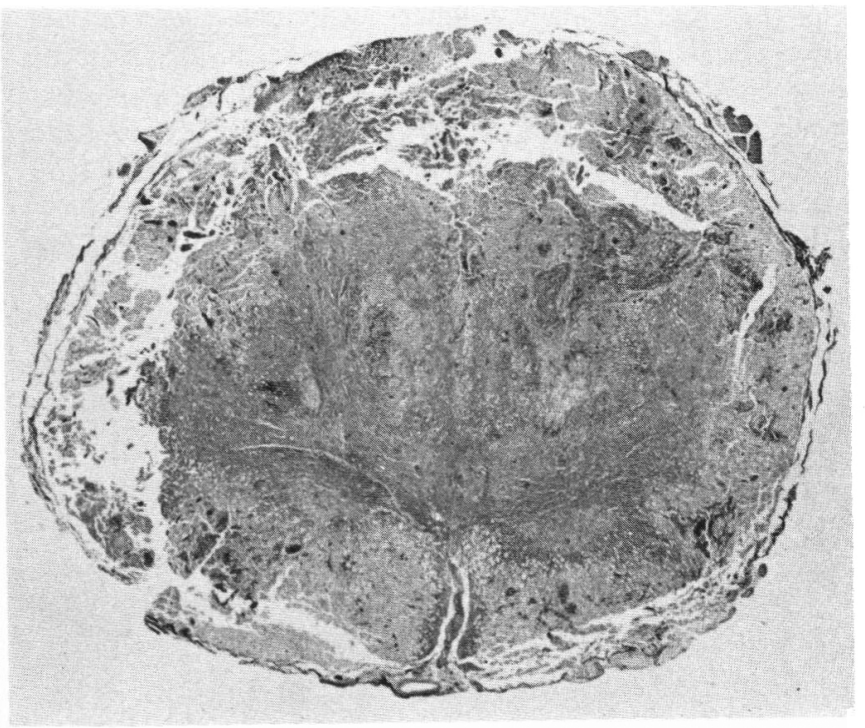

Figure 2 - Spinal cord at fourth thoracic level, showing obliteration of architecture by necrosis. The anterior funiculi and adjoining anterior horns of the ventral cord may be discerned as relatively intact but microscopic examination shows considerable vacuolization of the neuropil. The lumen of the anterior spinal artery is patent. (H\&E-LFB; $\mathrm{x} 7.5)$.

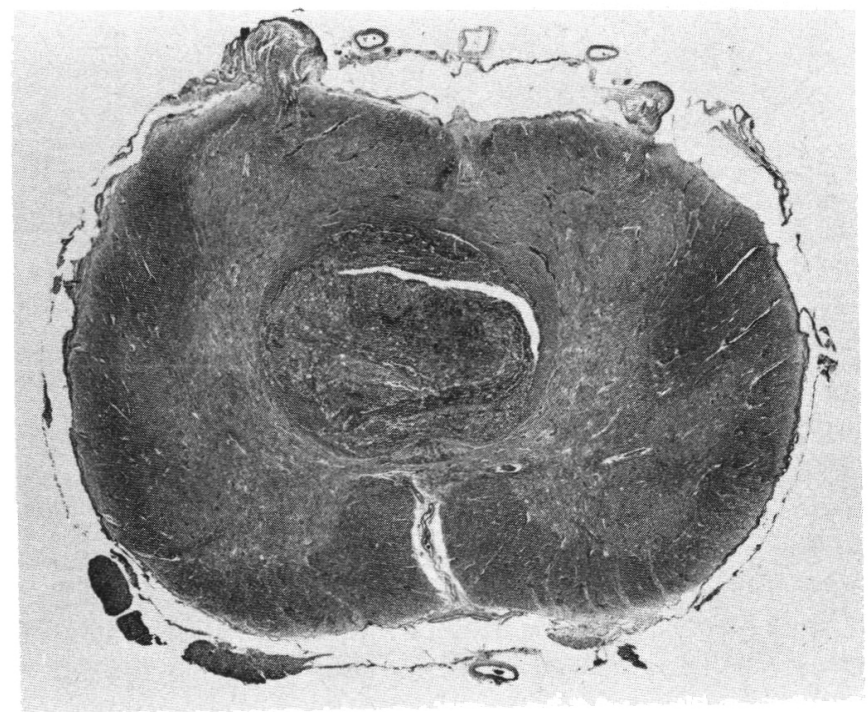

Figure 4-Spinal cord at first sacral segment, showing central necrosis. (H\&E-LFB; x 7.5). 
myelin were seen. In areas of necrosis more rostral or caudal to this level, polymorphonuclear cells were more abundant, suggesting these lesions were more recent. The tissue adjacent to areas of necrosis were edematous. In these zones the overall architecture was preserved but there were numerous cystic spaces, early breakdown of myelin associated with a nacrophage response, and axonal swellings.

A careful search for embolic material was negative. No occlusion of vessel lumen was observed in artery or vein at any level studied. Endothelial hyperplasia seen in capillaries adjacent to necrotic tissue appeared to be a reactive phenomenon. Perivascular and meningeal infiltrates of inflammatory cells were small and appeared to be secondary to tissue destruction (Figure 5). There was no evidence of vasculitis as a primary process in the areas free of necrosis. In cervical cord segments uninvolved by necrosis early Wallerian degeneration was observed in ascending tracts, with axonal swellings and macrophages containing myelin debris. An incidental finding was a small hydromyelia between $\mathrm{C} 6$ and $\mathrm{T} 7$.

In summary, several discrete zones of necrosis were found in the spinal cord extending from the $\mathrm{C} 8$ to $\mathrm{S} 2$ levels. The area of maximal necrosis straddled the vascular territories of the anterior and posterior spinal arteries. A preferred site of necrotic extension was the base of dorsal columns. No evidence of vascular occlusion was found.

Gross and microscopic studies of the cerebrum, brainstem and cerebellum were unremarkable.

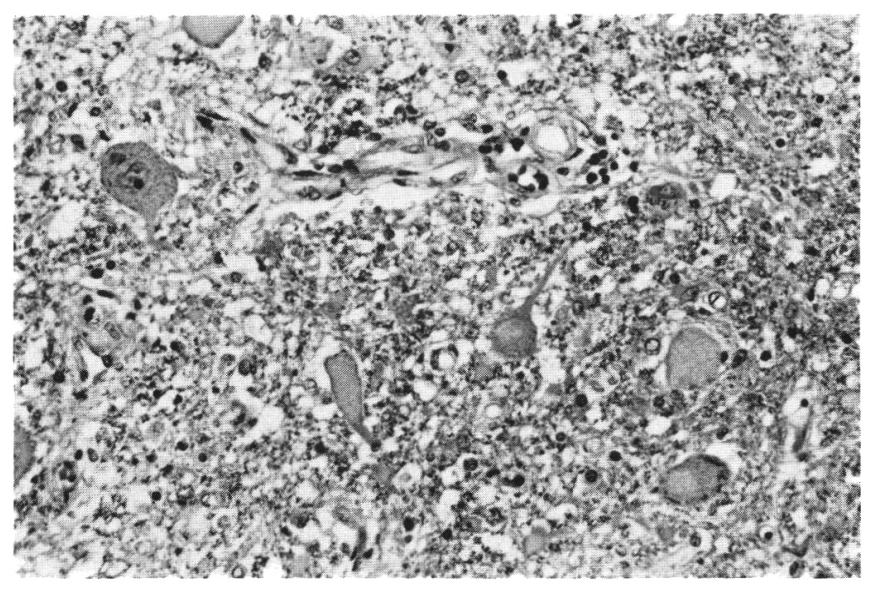

Figure 5 - Anterior horn at tenth thoracic spinal cord level showing perivascular lymphocytic infiltrates. The neurophil is vacuolated and the anterior horn cells show dispersal of the Nissl substance. (H\&E-LFB; x90).

\section{Discussion}

The case presented is that of a 79 year old man who developed spinal cord symptoms two weeks following resection of renal cell carcinoma. Examination showed a Brown-Sequard syndrome with a sensory level at T6. Pathologically the lesions are compatible with paraneoplastic subacute necrotic myelopathy. A Brown-Sequard syndrome is a known clinical presentation of this entity, as is a subacute progressive course, suggesting a propagating lesion (Case 26 - 1976).

Mancall and Rosales (1964) reported the findings in two cases of subacute necrotic myelopathy associated with malignancy and reviewed nine others. A review of published cases, (Grisold et al. 1980; Nishida and Ziegler 1973; Richter and Moore, 1968; Sieben et al. 1981; and Case 26 - 1976) and our present case indicates that males outnumbered females, in a ratio of 2 to 1 . The age of onset ranged from 16 to 79 years, the case of Grisold et al. (1980), being the youngest, and the present case the oldest. The median age of onset was 56 years, with all but three over the age of 40 years. The most common associated neoplasms were lung (5 cases) and lymphoproliferative malignancies (4). Others included sarcoma (1), breast (2), prostate, skin, stomach, thyroid, ovary and kidney (1 each). The duration of symptoms before death ranged from 5 days to 16 months. All but one patient died within 5 months, and the median survival was only 5 weeks.

In the reported cases, the number of spinal cord segments described as showing necrosis ranges from three to "entire cord"'. Despite some imprecise reports, the average number of segments involved can be estimated as 15 , an indication of the considerable rostral-caudal extent of these lesions. There was no correlation between duration of illness and extent of spinal cord lesions. At least part of the thoracic cord was involved in 16 of 18 cases, the cervical cord in 8 of 18 and lumbosacral cord in 12 of 18. Examination of cerebrospinal fluid was generally not helpful. The protein and cell count may be normal or elevated. In certain instances the albumino-cytologic dissociation of Guillain-Barré syndrome may be mimicked (Nishida and Ziegler 1973).

The etiology of paraneoplastic necrotic myelopathy remains obscure. The evidence thus far does not support a vascular etiology. The extent of necrosis is not limited to areas of anterior or posterior spinal artery supply (see Fig. 1). The arteries supplying the spinal cord have not demonstrated emboli, and the considerable rostral-caudal extent, as in the present case, may be larger than the territory supplied by any segmental artery. Vasculitis has been proposed as an underlying mechanism by Lester et al. (1979) who reported a case of a 66 year old man with Hodgkin's disease who developed a necrotic myelopathy. At autopsy two weeks after onset of symptoms intense vasculitis was found in both intact and necrotic spinal cord. These authors suggested that vasculitis caused necrosis which was missed in other reported cases of paraneoplastic subacute necrotic myelopathy because of resolution of vasculitis before death. However, our case, examined only three weeks after onset of symptoms, did not show vasculitis, and vasculitis as a primary process has not been reported in other cases, some of which died as early as five days after onset of illness (Mancall and Rosales 1964).

The present case did not reveal any systemic malignancy at the time of death. However, a visceral tumour was removed about two weeks prior to the onset of neurologic symptoms. Richter and Moore (1968) reported a case with lymphoma who developed necrotic myelopathy several months after the last chemotherapy treatment. No lymphoma was found at autopsy. Sieben et al. (1981) recently described an unusual case of a 56 year old female who developed subacute necrotic myelopathy eight years after cure of a breast carcinoma. The relationship to the previously diagnosed neoplasm is conjectural.

An interesting feature of the present case was the multifocal nature of the spinal cord lesion. Both Richter and Moore's cases were multifocal, with additional sites of involvement outside the spinal cord - in case 1 the optic nerve and chiasm, in case 2 the internal capsule and pons. Their case 2 was 
remarkable in having a biphasic illness. At autopsy both old static and newly necrotic lesions were found in the spinal cord. In Mathieson's case there was softening of medulla and pons (Mancall and Rosales 1964).

Any proposed pathogenesis for subacute necrotic myelopathy must account for its variable relationship to systemic cancer and its predilection for spinal cord with subacute progression and occasional multifocal involvement. Several authors have speculated on a possible remote effect of neoplasm on the metabolism of spinal cord (Mancall and Rosales 1964). Such an effect would depend on an interaction between presumed secretory products of the tumor and intrinsic qualities of the spinal cord which render it vulnerable to these products.

\section{REFERENCES}

Case records of the Massachusetts General Hospital - Case 26-(1976). N. Engl. J. Med. 294: 1147-1454.
Grisold, W., Lutz, D., Wolf, D. (1980). Necrotizing myelopathy associated with acute lymphoblastic leukemia. Acta Neuropathol. (Berl.) 49: 231-235.

Folliss, A.G.H., Netsky, M.G. Progressive necrotic myelopathy, in: Handbook of Clinical Neurology, vol. 9, Ed. P.J. Vinken, G.W. Bruyn, North Holland Publication Co., Amsterdam, 1970.

Lester, E.P., Feld, E., Kinzie, J.J., Wollman, R. (1979). Necrotizing myelopathy complicating Hodgkin's disease. Arch Neurol. 36: 583-585.

Mancall, E.L., Rosales, R.K. (1964). Necrotizing myelopathy associated with visceral carcinoma. Brain 87: 639-656.

Nishida, T., Ziegler, D.K. (1973). Acute necrotic myelopathy in association with lymphoma cells of the reticulo-endothelial system, acute torticollis, and recurrent cardiac arrest. Confin. Neurol. 35: 346-355.

Richter, R.B., Moore, R.Y. (1968). Non-invasive central nervous sytem disease associated with lymphoid tumours. Johns Hopkins Med. J. 122: 271-283.

Sieben, G.M., De Reuck, J.L., De Bruyne, J.C., Vander Eecken, H.M. (1981). Subacute necrotic myelopathy. Its appearance eight years after cure of a breast carcinoma. Arch. Neurol. 38: 775-777. 